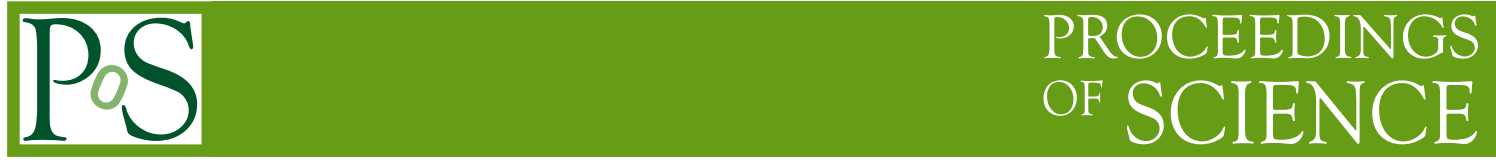

\title{
Microquasars: Progress made and open questions
}

\author{
I.F. Mirabel ${ }^{* \dagger}$ \\ European Southern Observatory. Alonso de Cordova 3107. Santiago, CHILE \\ E-mail: fmirabeldeso.org
}

In the last talk of the conference I summarized the main progress and contributions to high energy astrophysics made by studies of microquasars in our Galaxy. To stimulate the general discussion I have underlined some of the questions that will guide in the near future the research in this area of astrophysics. Here I present the viewgraphs and questions formulated during the general discussion.

VI Microquasar Workshop: Microquasars and Beyond September 18-22 2006

Società del Casino, Como, Italy

\footnotetext{
${ }^{*}$ Speaker.

† On leave from CEA-Saclay. France
} 

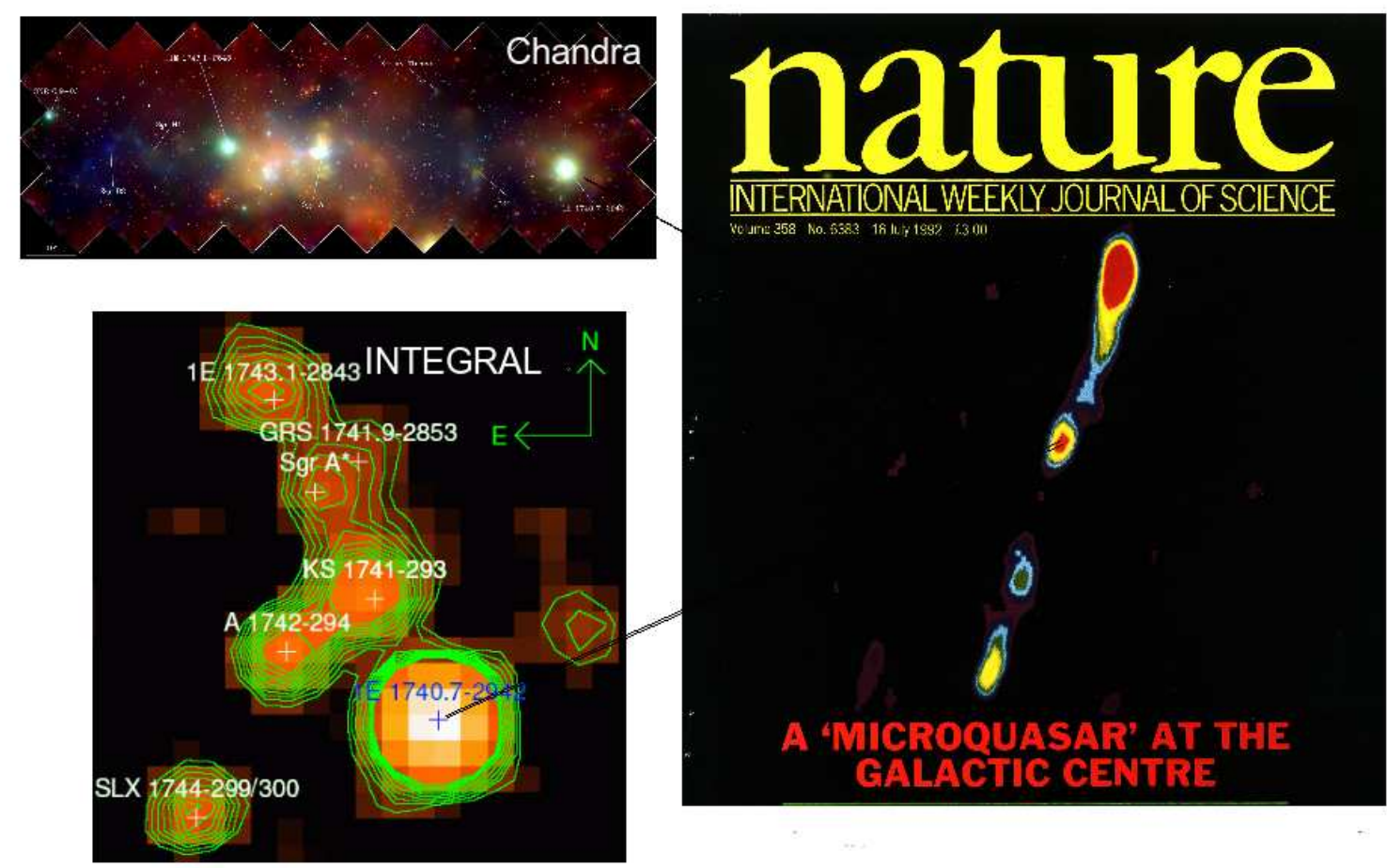

Figure 1: Chandra and INTEGRAL images of the Galactic Center region on the left. On the right is shown the $\lambda 6 \mathrm{~cm}$ compact counterpart of the high energy source at the center of collimated jets that are the trace of synchrotron electrons and possible positrons streaming away from the compact source up to distance of a few light years.

\section{The microquasar 1E 1740.7-2942 still a mystery}

Figure 1 shows the Chandra and INTEGRAL images of the Galactic Center region, where the most luminous source in the hard X rays is the famous Einstein source 1E 1740.7-2942, which is a source of relativistic jets that extend up to a few light years. The flux, spectrum shape and time variability in the X-rays are similar to those that would be observed from Cygnus X-1 located in the Galactic Center region, which supports the hypothesis that 1E 1740.7-2942 is a stellar-mass black hole binary. However, until today it has not been found -even using the VLT in the near and mid-infrared-, an unambiguous optical or infrared counterpart of the donor star in this putative black hole binary.

\section{Gamma rays from compact binaries: microquasar jets or pulsar winds ?}

Recent observations have shown that some compact stellar binaries radiate the highest energy light in the universe. The challenge has been to determine the nature of the compact object and whether the very high energy gamma rays are ultimately powered by pulsar winds or relativistic jets (Figure 2).

Multiwavelength observations have shown that two (PSR B1259-63 and LS I +61 303) of the three gamma-ray binaries known so far are neutron star binaries and that the very energetic gamma- 

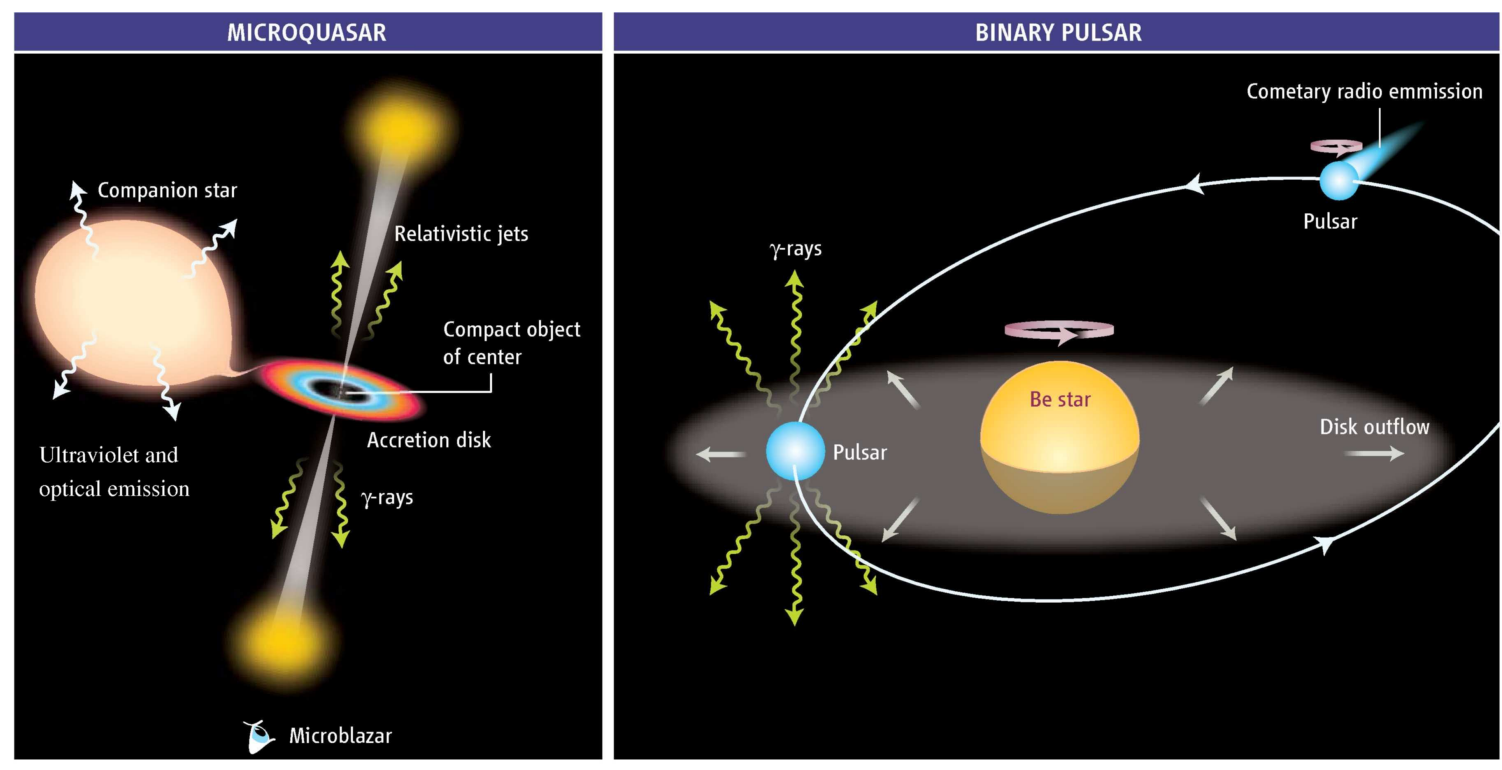

Figure 2: Alternative models for very energetic gamma-ray binaries. Left: Microquasars are powered by compact objects (neutron stars or stellar-mass black holes) via mass accretion from a companion star. The jets boost the energy of stellar winds to the range of very energetic gamma-rays. Right: Pulsar winds are powered by rotation of neutron stars; the wind flows away to large distances in a comet-shape tail, as has been shown in to be the case for LS I +61 303. Interaction of this wind with the companion-star outflow may produce very energetic gamma-rays.

rays from these two sources may be produced by the interaction of pulsar winds with the wind from the companion star. In fact, in this meeting were presented observations at radio wavelengths that support the idea that LS I +61 303 may be a gamma-ray pulsar rather than a microquasar. As expected from the pulsar wind model (right panel of Fig. 2), VLBA images of the radio emission show a relativistic wind from the compact object that spins as a function of the orbital phase. However, no pulsations have so far been detected from LS I +61 303.

At this time it is an open question whether the third gamma-ray source (LS 5039) is also powered by a pulsar wind or a microquasar jet, where relativistic particles in collimated jets would boost the energy of the wind from the stellar companion to TeV energies. High resolution maps at radio wavelengths would tell whether the direction of the jets spin as a function of the orbital phase or their position angle in the sky remain the same. On the other hand, it would be interesting a search for $\mathrm{TeV}$ emission from confirmed black hole binaries, which would provide support to the microquasar jet mechanism for the production of very energetic gamma-rays.

These $\mathrm{TeV}$ binaries do not require Doppler boosting, namely, they are not microblazars.

\section{Perspectives to determine the spin of black holes}

Three methods have been proposed to determine the spin of black holes. They are based on measurements of: 1) the temperature of the continuum spectrum. Due to the shrink of the inner accretion disk in rapidly rotating (Kerr) black holes, the temperature increases with angular momentum. 2) the Fe line at $6.7 \mathrm{keV}$, which is skewed towards lower energies as a function of the 

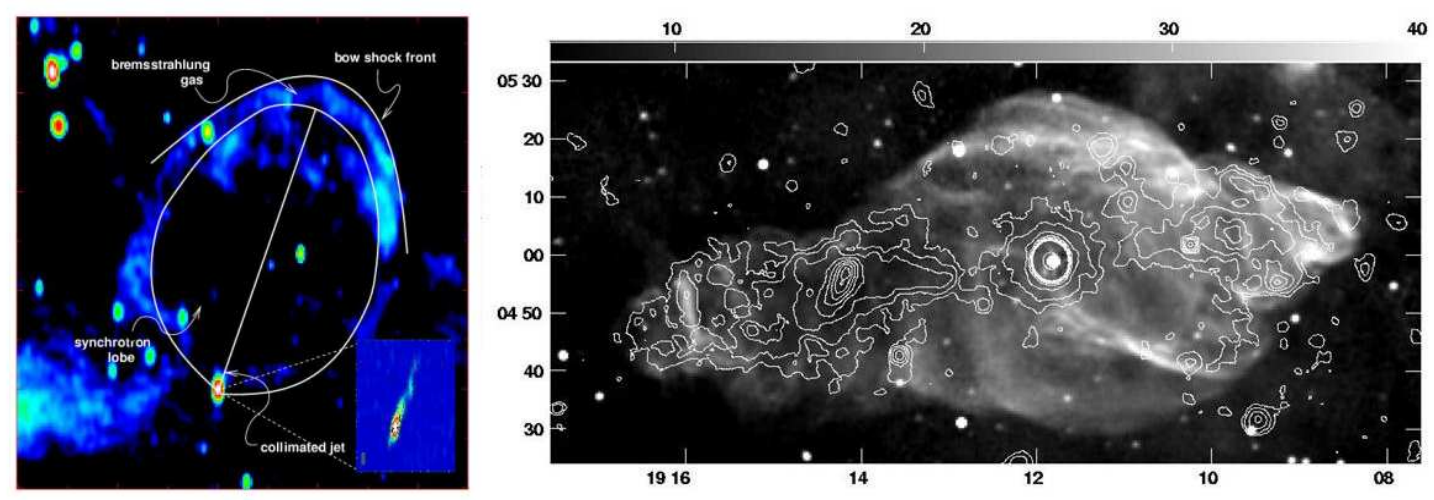

Figure 3: Left panel: Jet-powered nebula around Cygnus X-1 from Gallo et al.(2005). Right panel: Image at $\lambda 20 \mathrm{~cm}$ of the nebula W50 that hosts SS 433, from Dubner et al. (1998).

angular momentum. 3) the maximum fix frequency of quasi-periodic oscillations (QPOs), which is a function of the basic parameters that define astrophysical black holes, namely, the mass and the spin.

In this meeting new exciting insights on the spin of black holes were presented. Refining the analysis of the continuum spectrum it was found that GRS 1915+105 contains a black hole spinning near the maximum rate, whereas GRO J1655-40, LMC X-3, and 4U 1543-47 have moderate spins. It is interesting that the source with maximum spin is also one of the most powerful jet sources. However, due to the small number statistics it is still an open question whether the power of relativistic jets is a function of the spin, as predicted by different models.

In this field there are still the following basic caveats:

a) The physics of QPOs is still not fully understood. In this meeting it has been proposed that they are due to global modes that arise as spontaneous instabilities rather than as orbiting "blobs".

b) It is still uncertain how the forest of warm absorber lines may affect the X-ray continuum, and therefore the estimates of the temperature of the inner accretion disk.

\section{Dark outflows and super-Eddington sources}

In last years there have been increasing evidences showing that relativistic and non-relativistic mass outflows from accreting compact objects may be radiatively inefficient. The evidences reside mostly in the interaction of the jets with the interstellar environment. They are: 1) The moving X-ray jets from the microquasars XTE J1550-564 and H1743-322 that produced in real time synchrotron bow shocks far away from the sources and long after X-ray outbursts from the compact binary, and 2) The large scale interstellar structures inflated by the relativistic jets as observed at radio continuum wavelengths in Cygnus X-1 and SS 433 (Figure 3).

In SS 433 a mechanical luminosity of the relativistic jets of $\geq 10^{39} \mathrm{erg} \mathrm{s}^{-1}$ has been estimated from spectral lines of ions of $\mathrm{H}, \mathrm{He}$, and Fe at optical, infrared and X-ray wavelengths. In SS 433 more than $50 \%$ of the energy is not radiated and it has been estimated that more than $30 \%$ of the accreted mass is being ejected in the form of massive winds and relativistic jets. Clearly, SS 433 is a super-Eddington source of stellar mass with anysotropic outflow and radiation that from other 

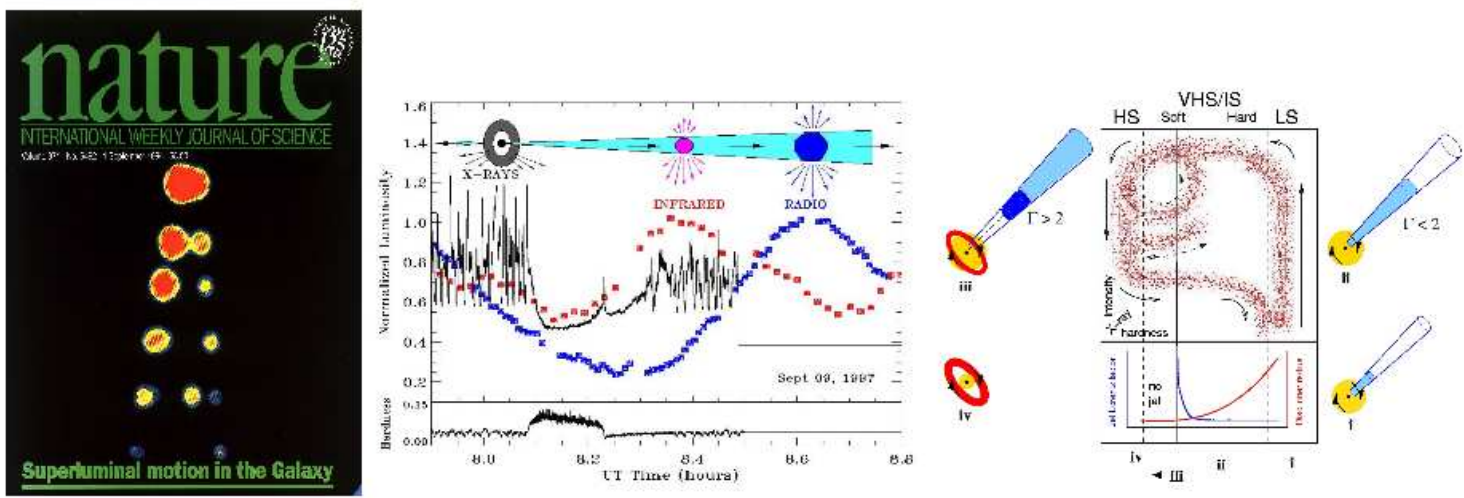

Figure 4: Left panel: Superluminal ejection in GRS 1915+105; central panel: multiwavelength simultaneous observations of instabilities in the accretion disk and the genesis of jets; Right panel: Unified model for black hole accretion-jet phenomena (Fender et al 2004).

viewing angle would be classified as an ultraluminous X-ray source (ULX). It may be used as a probe of ULX's in external galaxies.

The following questions remain open:

1) What is the relation between massive winds and warm absorbers ?

2) If black holes of intermediate mass $\left(\geq 100 \mathrm{M}_{\odot}\right)$ are prolific, why no one has been identified in the Milky Way or the Magellanic Clouds, where the dynamical mass of compact objects in binaries can be determined?

\section{Disk-jet coupling}

In 1994 it was observed a mayor ejection event from GRS 1915+105 that was followed simultaneously with BATSE on the Compton Observatory and the Very Large Array (Figure 4, left panel). The connection between the inner disk-corona and the jets became clear because the ejection took place at the time of a sudden drop in the 20-100 keV flux. At that time it was assumed that the high energy electrons producing the hard X-ray radiation had been suddenly blown away in the form of collimated relativistic jets. The physical nature of the instability that produced such transition remains unknown.

Four years later was obtain further insight into the connection between accretion disk instabilities and the formation of jets. In $\sim 1$ hour of simultaneous multiwavelength observations of GRS 1915+105 during the frequently observed 30-40 min X-ray oscillations in this source, the connection between sudden drops of the x-ray flux from the accretion disk and the onset of jets was observed again in several occasions (Figure 4, central panel). The triggers of jets are instabilities in the accretion disk (transition from low-hard to high-soft states). The X-ray spike in the central panel marks the onset of a shock through a compact, steady jet.

A comprehensive phenomenological model based on the notion that the jet phenomenology is part of the accretion process was later proposed by Fender et al (2004; Figure 4 right panel), to which I refer the reader for a detailed description. Analogous transitions have now been observed in 
the quasars 3C120, 3C279 and 3C390, the diagram in the right panel of Figure 4 being considered in observations of the disk-jet coupling in accreting black holes of all mass scales.

\section{The black hole at the Galactic Center}

European and American teams have been studying the kinematics of the central cluster of massive stars with the VLT and Keck respectively, where using adaptive optics at infrared wavelengths from the ground becomes competitive with space astronomy. Both teams reach similar results and estimate a black hole mass of $3 \times 10^{6} \mathrm{M}_{\odot}$. However, there are following puzzles:

1) How could a massive cluster of massive stars be formed and survive in a region of 10 light years radius from the supermassive black hole?

2) Observing with the VLT, QPOs of about 15 min were reported. However, no such QPOs have been observed so far with Keck. In microquasars it is known that QPOs of maximum fix frequency are only observed sporadically but not permanently. Could the presence and absence of these QPOs be due to chance ?

3) The flares in Sgr A* exhibit similar time delays between the X-rays, infrared and radio wavelengths as observed in the flares of GRS 1915+105 (Figure 4, central panel). These time delays are comparable, irrespective of the mass of the black holes which indicates that the radiating plasma has detached from the gravitational field of the black hole.

\section{Microquasars and beyond}

In the three astrophysical manifestations of astrophysical black holes are found the same three basic ingredients: a black hole, an accretion disk, and relativistic jets (Figure 5).

For microquasars, quasars and GRBs have been proposed the same alternative models:

Jet models: Discontinuous ejections of blobs (internal shock model) versus pointing flux.

Two flow model: collimated MHD flow plus super-relativistic pair beamed jet

Jet production models: MHD centripetal (inner) plus magnetic dissipation at larger scales.

\section{Microquasar - GRB connections}

In previous publications I had proposed that microquasars in our Galaxy can be used as nearby probes of the collapsar model for the formation of stellar-mass black holes. In particular, the kinematics of microquasars can be used to constrain the energy of natal explosions during the formation of black holes. For instance, from the chemical composition found in the atmosphere of the donor star and runaway velocity of GRO J1655-40 it was proposed that the black hole in this microquasar was formed through a very energetic supernova.

The question on whether all black holes form through energetic explosions or some form by direct collapse is related to the question on whether all long GRBs are associated with hypernovae (type Ib/c). The recent observations of GRB 060614 by Della Valle et al. (2006) show that some long-lasting GRBs can be associated with very faint supernova. This result is consistent with the finding that the black holes of $\geq 10 \mathrm{M}_{\odot}$ in Cygnus X-1 and GRS 1915+105 have not receive a large linear momentum during formation (Figure 6). 


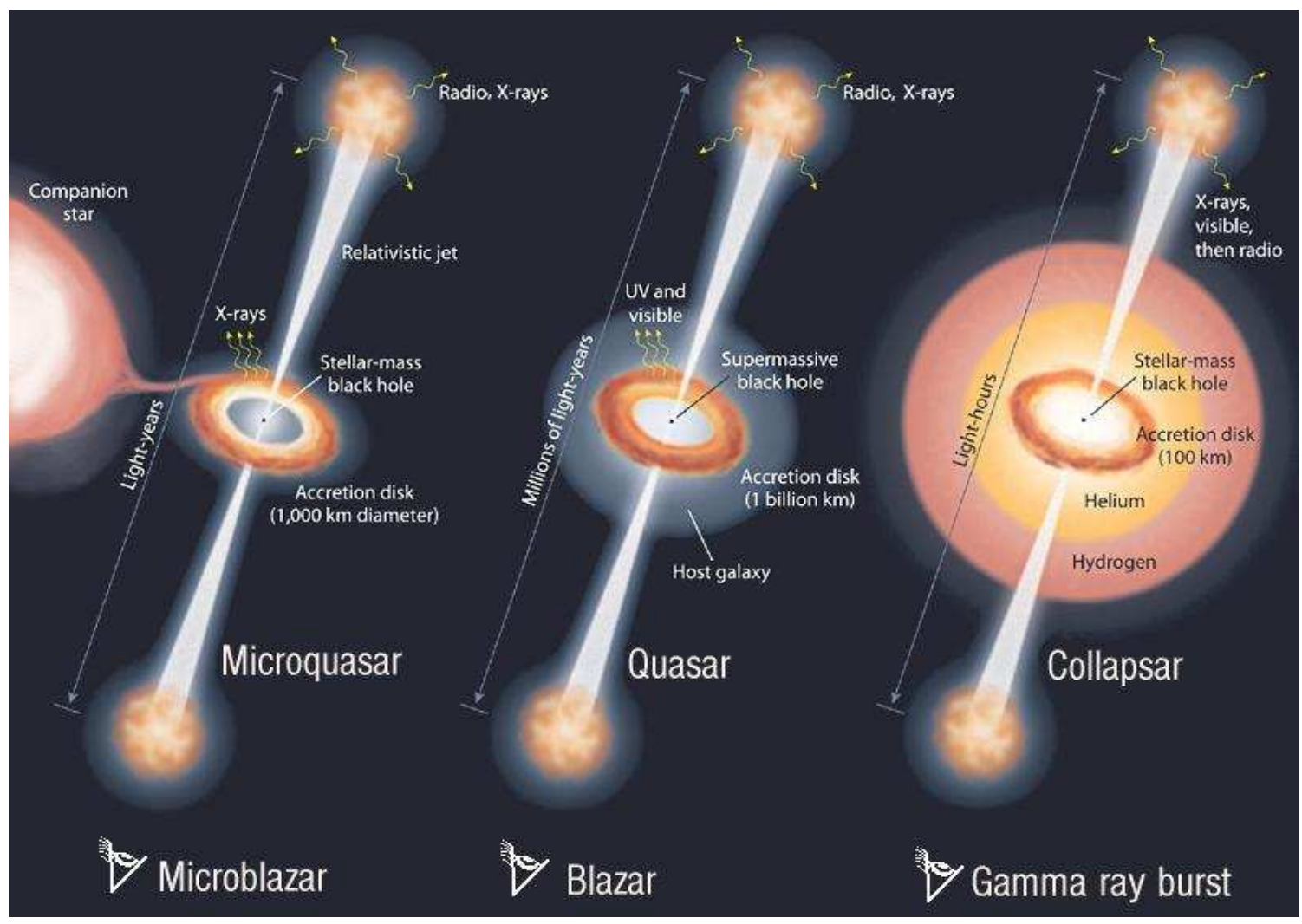

Figure 5: The three manifestations of astrophysical black holes.
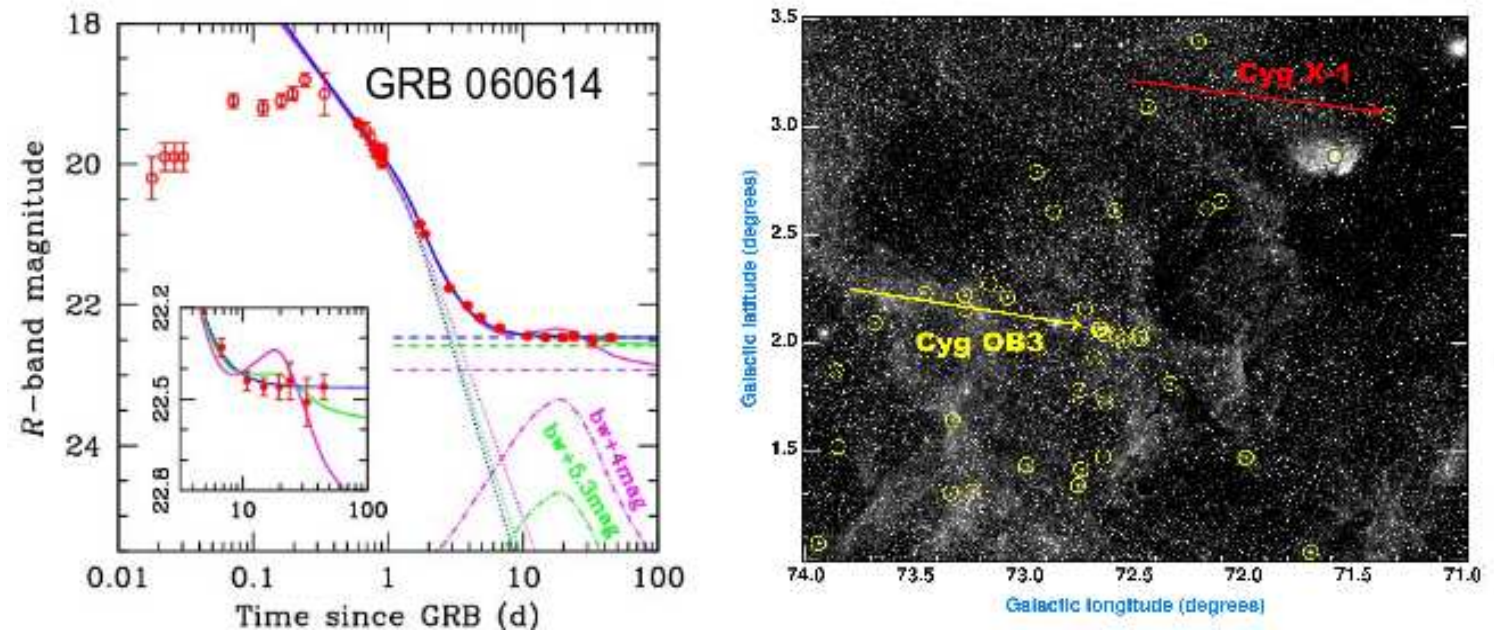

Figure 6: Left panel: R-band light curve of the GRB 060614 afterglow showing no luminous supernova (Della Valle et al. 2006). Right panel: Proper motion of of Cygnus X-1 and the parent association of massive stars Cygnus OB3 showing that the microquasar remained at its birth place and did not received a strong kick. 


\section{Empirical correlations and analogies between stellar and black hole astrophysics}

Different empirical correlations between the X-ray and radio fluxes, noise spectrum, and QPOs as a function of black hole mass have been proposed. If these empirical correlations become more robust, independently of the models, the mass and spin of black holes will be determined. As in stellar astrophysics, in black hole astrophysics the luminosity is a function of size and temperature.

At present, black hole astrophysics is in an analogous situation as was stellar astrophysics in the first decades of the XX century. At that time, well before reaching the physical understanding of the interior of stars and the way by which they produce and radiate their energy, empirical correlations such as the HR diagram were found and used to derive fundamental properties of the stars, such as their mass. Analogous approaches are taking place in black hole astrophysics. Using correlations among observables such as the radiated fluxes in x-rays and radio waves, quasi-periodic oscillations, flickering frequencies, etc, fundamental parameters that describe astrophysical black holes such as the mass and spin of the black holes are being derived.

\section{References}

[1] Della Valle,M. et al. 2006, Nature 444, 1050

[2] Dubner, G. et al. (1998), Astron. J. 116, 1842

[3] Fender, R. et al. (2004), MNRAS 355, 1105

[4] Gallo, E. et al.(2005), Nature 436, 819

[5] Merloni, A. et al. (2003) MNRAS, 345, 1057 